J. Klin. Endokrinol. Stoffw. 2021 - 14:55-61 https://doi.org/10.1007/s41969-021-00133-0 Angenommen: 25. März 2021 Online publiziert: 26. April 2021 (๑) Der/die Autor(en) 2021

\section{Christian Muschitz ${ }^{1,2}$}

'Externe Lehre, Medizinische Universität Wien, Wien, Österreich

${ }^{2}$ II. Medizinische Abteilung, Krankenhaus der Barmherzigen Schwestern Wien, Wien, Österreich

\title{
Antikörper-Therapie bei Osteoporose: Mechanismen, Therapieziele und Outcomes
}

\section{Die Größe des Problems}

In Österreich erlitten im Jahr 2018 mehr als 93.000 Patientinnen und Patienten im Alter $50+$ eine niedrigtraumatische (= osteoporotische) Fraktur. Diese Frakturen traten zu etwa zwei Dritteln bei Frauen auf. Ab Ende der 50. Lebensdekade nimmt der Anteil dieser Frakturen bei beiden Geschlechtern mit steigendem Alter kontinuierlich zu. Ab der achten Lebensdekade beträgt der Anteil dieser Frakturen in der weiblichen Population mehr als $70 \%$ und etwa $65 \%$ in der männlichen Population. Die Kosten für die stationäre und ambulante Erstversorgung dieser Frakturen exklusive Rehabilitation betrugen allein im Jahr 2018 mehr als 160 Mio. $€$ [1].

\section{Grundlagen der Osteoporose- therapie}

Das Ziel jeder osteologischen Behandlung ist die Senkung eines erhöhten Frakturrisikos und somit die Verhinderung einer osteoporotischen Fraktur bzw. im Fall einer prävalenten (Index-)Fraktur die Verhinderung einer konsekutiven Fraktur.

Die Basis der Therapie ist im Vorfeld immer ein ausgeglichener Kalziumstoffwechsel und ein adäquater VitaminD-Spiegel (25-OH-Vitamin-D-Spiegel $>20 \mathrm{ng} / \mathrm{ml} \mathrm{bzw}$. $50 \mathrm{nmol} / \mathrm{l})$. Das früher gültige Dogma „je höher, desto besser" ist nicht mehr gültig. Die primäre Quelle des Kalziums sollte die Nahrung sein, eine orale Supplementation - sofern indiziert - sollte $500 \mathrm{mg} / \mathrm{d}$ nicht überschreiten.
Osteoporosemedikamente können nach ihrer Wirkung in zwei Gruppen eingeteilt werden:

- Antiresorptive (antikatabole) Me-

dikamente hemmen primär den

Osteoklasten. In diese Gruppe fallen

Bisphosphonate (oral, i.v.), SERMs

(selektive Östrogen-Rezeptor-Modu-

latoren) und Denosumab.

- Anabole Medikamente aktivieren primär Osteoblasten. Vertreter dieser Substanzgruppe sind Teriparatid (1-34 Parathormon) und Romosozumab [2].

\section{Denosumab}

\section{Wirkprinzip}

Denosumab (Prolia ${ }^{\circledR}$ ) ist ein humaner monoklonaler IgG2-Antikörper mit einer Molekülmasse von $147 \mathrm{kDa}$, dessen Effekt auf der Bindung an das Protein
RANKL (Receptor Activator of NF- $\kappa \mathrm{B}$ Ligand) beruht. Dieses ist für die Bildung, die Funktion und das Überleben der Osteoklasten wichtig. Durch die Hemmung der Wechselwirkung von RANKL mit seinem Rezeptor RANK (Receptor Activator of NF- $\kappa B$ ) auf der Oberfläche von Osteoklasten wird die Reifung und Aktivierung dieser Zellreihe rasch supprimiert. Konsekutiv wird die Knochenresorption vermindert, es erhöhen sich so die Masse und die biomechanischen Eigenschaften des Knochens. Die in der Osteoporose zugelassene Dosierung beträgt $60 \mathrm{mg}$ subkutan, die Dauer der Wirkung beträgt sechs Monate. Aufgrund der Pharmakokinetik ist - im Gegensatz zu langwirksamen Bisphosphonaten - das Applikationsintervall zeitlich genau einzuhalten. Denosumab ist auch in der Onkologie in höherer Dosierung unter dem Namen Xgeva ${ }^{\circledR}$ im Einsatz.

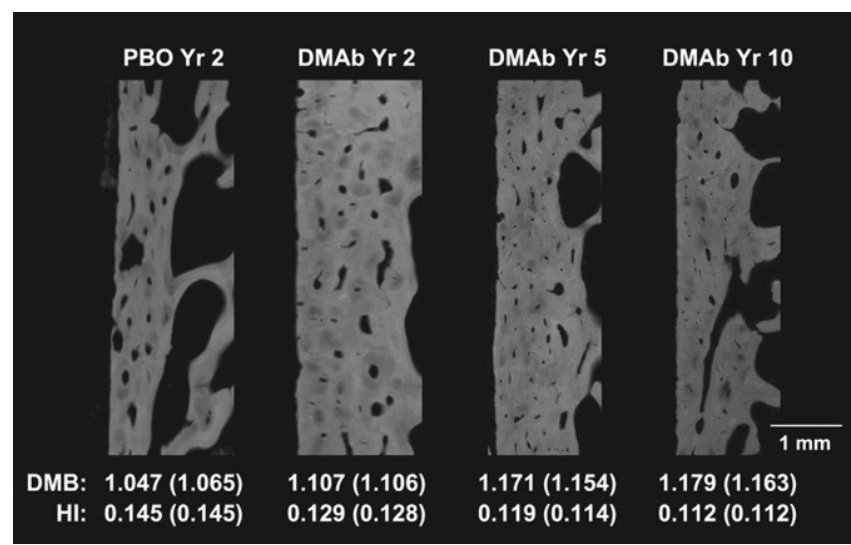

Abb. 1 A Transiliakale Biopsien unter mehrjähriger Therapie mit Denosumab (DMab) vs. Placebo (PBO). DMB degree of mineralized bone, HI heterogeneity index. (Aus [3]. ๑ 2018 Endocrine Society, https://creativecommons.org/licenses/by/4.0/. Diese Abbildung fällt nicht unter die Creative Commons CC BY-Lizenz dieser Publikation) 


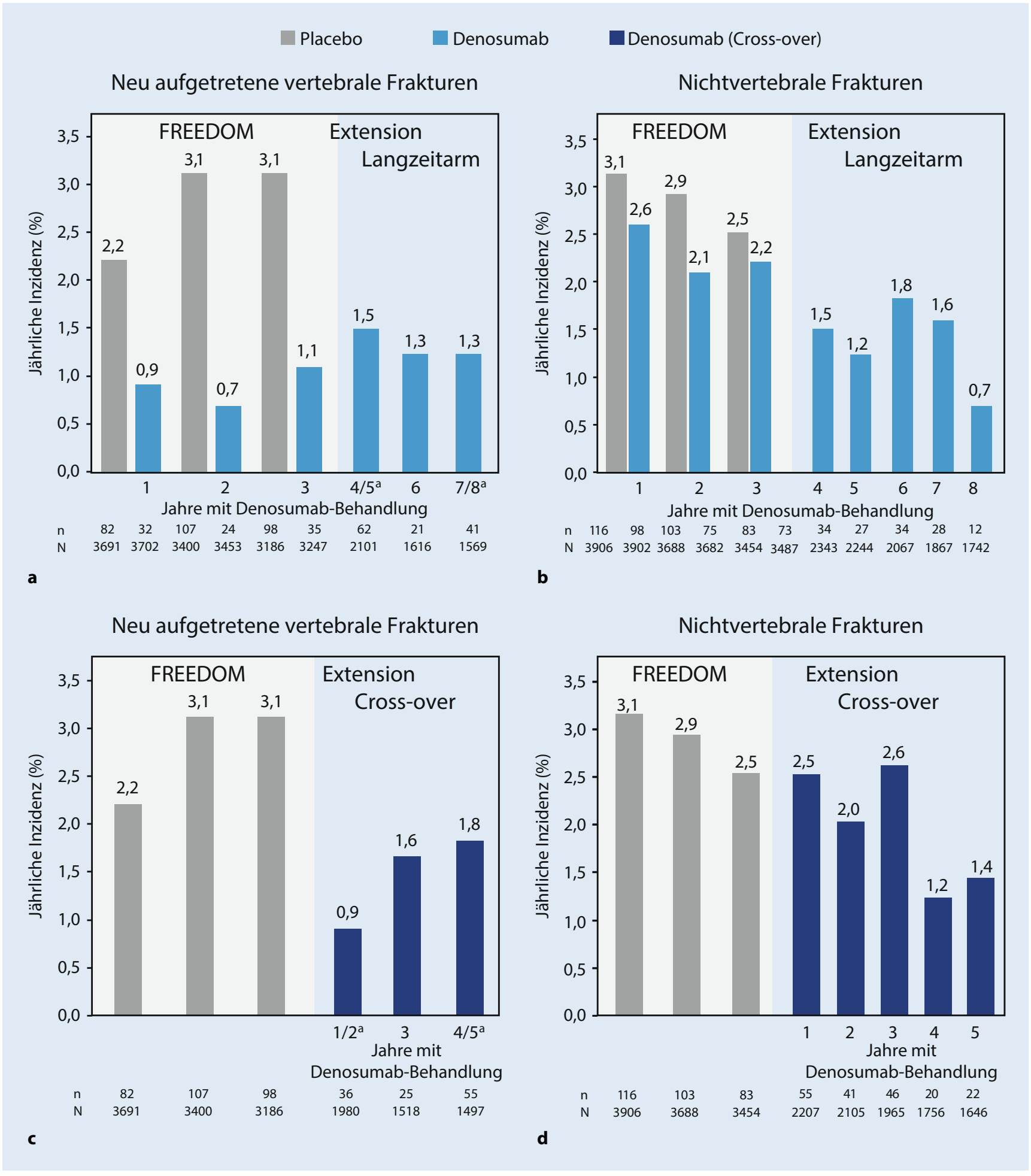

Abb. $2 \Delta$ Verringerung der osteoporotischen Frakturen unter Denosumab. Die FREEDOM-Studie war in den Jahren 1-3 Placebo-kontrolliert. Der Langzeitarm (Extension) umfasst Patientinnen mit Denosumab von Beginn an. Patientinnen mit Placebo wurden im vierten Jahr der Studie umgestellt (Cross-over-Arm). $n=$ Anzahl Frauen mit $\geq 1$ Fraktur; $N=$ Anzahl Frauen, bei denen im Beobachtungszeitraum eine Röntgen-Evaluation der Wirbelsäule durchgeführt wurde (vertebrale Frakturen) bzw. Anzahl Frauen, die zu Beginn jedes Beobachtungszeitraums noch an der Studie teilnahmen (nichtvertebrale Frakturen); ${ }^{a}$ Ein-Jahres-Inzidenz (2-Jahres-Inzidenz/2). (Mit Genehmigung aus [5]. ๑ The Author(s) $2015 \mathrm{http} / / /$ creativecommons.org/ licenses/by-nc/4.0/. Diese Abbildung fälltt nicht unter die Creative Commons CC BY-Lizenz dieser Publikation) 
Hier steht eine Anzeige.

\section{曾 Springer}




\section{Daten aus Biopsien}

Daten aus Knochenbiopsien über einen Zeitraum von zehn Jahren Therapie mit Denosumab zeigten eine normale Histologie des Knochens. Die Histomorphometrie ergab eine normale Knochenstruktur und einen - der Wirkung des Antikörpers geschuldeten - verringerten Knochenumbau (Remodeling). Der Effekt im Jahr zehn war vergleichbar mit den Jahren zwei/drei oder fünf. Der Grad der Mineralisierung wurde erhöht, und die Heterogenität konnte im direkten Vergleich zu Placebo vermindert werden. Die Veränderung bzw. die Verbesserung der Mineralisierung war am stärksten in den ersten drei bis fünf Jahren der Therapie, danach wurde unter Therapie ein Gleichgewicht gehalten. Es wurden unter Langzeittherapie keine Hinweise auf eine Osteomalazie, eine Knochenmarksfibrose oder einen atypisch konfigurierten Knochen gefunden (• Abb. 1; [3]).

\section{Effizienz der Frakturreduktion}

Bei der Behandlung der postmenopausalen Osteoporose ist eine Reduktion von vertebralen und nichtvertebralen Frakturen inklusive proximaler Femurfrakturen in Studien bis zu 10 Jahre nachgewiesen. Die Wirkung ist unabhängig von einer eventuellen Vorbehandlung mit Bisphosphonaten [4]. Die Behandlungsdauer ist unklar, bei entsprechender Indikation ist Denosumab als Langzeittherapie einzusetzen.

Die längste Studie bei postmenopausalen Frauen mit Osteoporose und einem erhöhten Knochenbruchrisiko war FREEDOM. Die ersten drei Jahre waren placebokontrolliert, in weiteren sieben Jahre waren alle Patientinnen unter Therapie. Die Therapie mit Denosumab führt $\mathrm{zu}$ einer raschen und konstant anhaltenden Reduktion des Frakturrisikos für vertebrale, nonvertebrale und Hüftfrakturen in den publizierten 8-Jahres-Daten (• Abb. 2). Korrespondierend verbessert sich die Knochenmineraldichte kontinuierlich, und die serologischen Marker für die Aktivität von Osteoklasten bleiben auf einem konstant niedrigen Niveau (• Abb. 3; [5]).
Nach Absetzen von Denosumab scheint es im Gegensatz zu den Bisphosphonaten zu einem raschen Anstieg des Knochenumbaus und in weiterer Folge zu einer Abnahme der Knochenmineraldichte $\mathrm{zu}$ kommen. Dieses als „Rebound-Effekt" bezeichnete Phänomen kann auch zu Frakturen kurze Zeit nach Absetzen von Denosumab führen und bedarf einer engmaschigen Kontrolle im Verlauf. Kiefernekrosen und atypische Femurfrakturen sind bei dieser für Osteoporose zugelassenen Therapie eine sehr seltene Nebenwirkung mit einer Wahrscheinlichkeit $<0,1 \%[2,6]$.

Denosumab (gelbe Box RE1) ist gemäß Regeltext für folgende Krankheitsbilder zugelassen: Patientinnen mit Knochenbruchkrankheit (postmenopausale Osteoporose) mit hohem Frakturrisiko oder vorhergegangenen Frakturen nach inadäquatem Trauma, bei denen eine Therapie mit oralen Bisphosphonaten nicht möglich ist. Eine weitere Indikation ist die Behandlung der Osteoporose bei Männern mit erhöhtem Frakturrisiko. Da der Antikörper nicht renal eliminiert wird, ist Denosumab eine gute Option bei Niereninsuffizienz. Weitere mögliche Pathologien umfassen die Glukokortikoid-induzierte Osteoporose.

\section{Romosozumab}

\section{Wirkprinzip}

Romosozumab (Evenity ${ }^{\circledR}$ ) ist ein humanisierter monoklonaler Ig2-Antikörper mit einer Molekülmasse von $149 \mathrm{kDa}$. Der Antikörper fördert den Knochenaufbau und hemmt in einem geringeren Ausmaß zusätzlich den Knochenabbau. Die Effekte beruhen auf der Hemmung des Glykoproteins Sklerostin, das von Osteozyten insbesondere bei Immobilität gebildet wird und die Funktion, die Differenzierung, die Proliferation und das Überleben der Osteoblasten hemmt. Sklerostin übt seine Effekte durch Bindung an Rezeptoren auf der Oberfläche von Osteoblasten aus. Vereinfacht gesagt kommt es zu einer Hemmung der körpereigenen Hemmung von Osteoblasten [7].
J. Klin. Endokrinol. Stoffw. $2021 \cdot 14$ $55-61$

https://doi.org/10.1007/s41969-021-00133-0 (c) Der/die Autor(en) 2021

\section{Muschitz}

\section{Antikörper-Therapie bei Osteoporose: Mechanismen, Therapieziele und Outcomes}

\section{Zusammenfassung}

Antikörper zur Behandlung von Patientinnen und Patienten mit Osteoporose und einem erhöhten Knochenbruchrisiko sind effizient, sicher und einfach in der Anwendung. Für Denosumab liegen wissenschaftliche Daten über einen Beobachtungszeitraum von zehn Jahren vor. Romosozumab ist eine potente, rasch wirksame Behandlungsoption für Hochrisiko-Patienten.

Schlüsselwörter

Osteoporose - Denosumab - Romosozumab . Fraktur $\cdot$ Knochendichte

\section{Antibody therapy for osteoporosis: mechanisms, therapy goals, and outcomes}

\section{Abstract}

Antibodies for the treatment of patients with osteoporosis and an increased risk of bone fractures are efficient, safe, and easy to use. For denosumab, scientific data are available over an observation period of ten years. Romosozumab is a potent, rapidly effective treatment option for high-risk patients.

Keywords

Osteoporosis · Denosumab · Romosozumab . Fracture $\cdot$ Bone density

\section{Therapiedauer und Dosierung}

Die Therapiedauer mit Romosozumab beträgt zwölf Monate. Der Antiköper wird einmal monatlich in einer Dosierung von $210 \mathrm{mg}$ (zwei Injektionen zu $105 \mathrm{mg}$ ) subkutan verabreicht. Im Anschluss an diese Therapie ist - so wie nach einer anabolen Therapie mit Teriparatid - eine mehrjährige antiresorptive Konsolidierungstherapie notwendig.

\section{Wirkung auf Knochendichte und Frakturrisiko}

In der placebokontrollierten FRAMEStudie bei postmenopausalen Frauen mit 


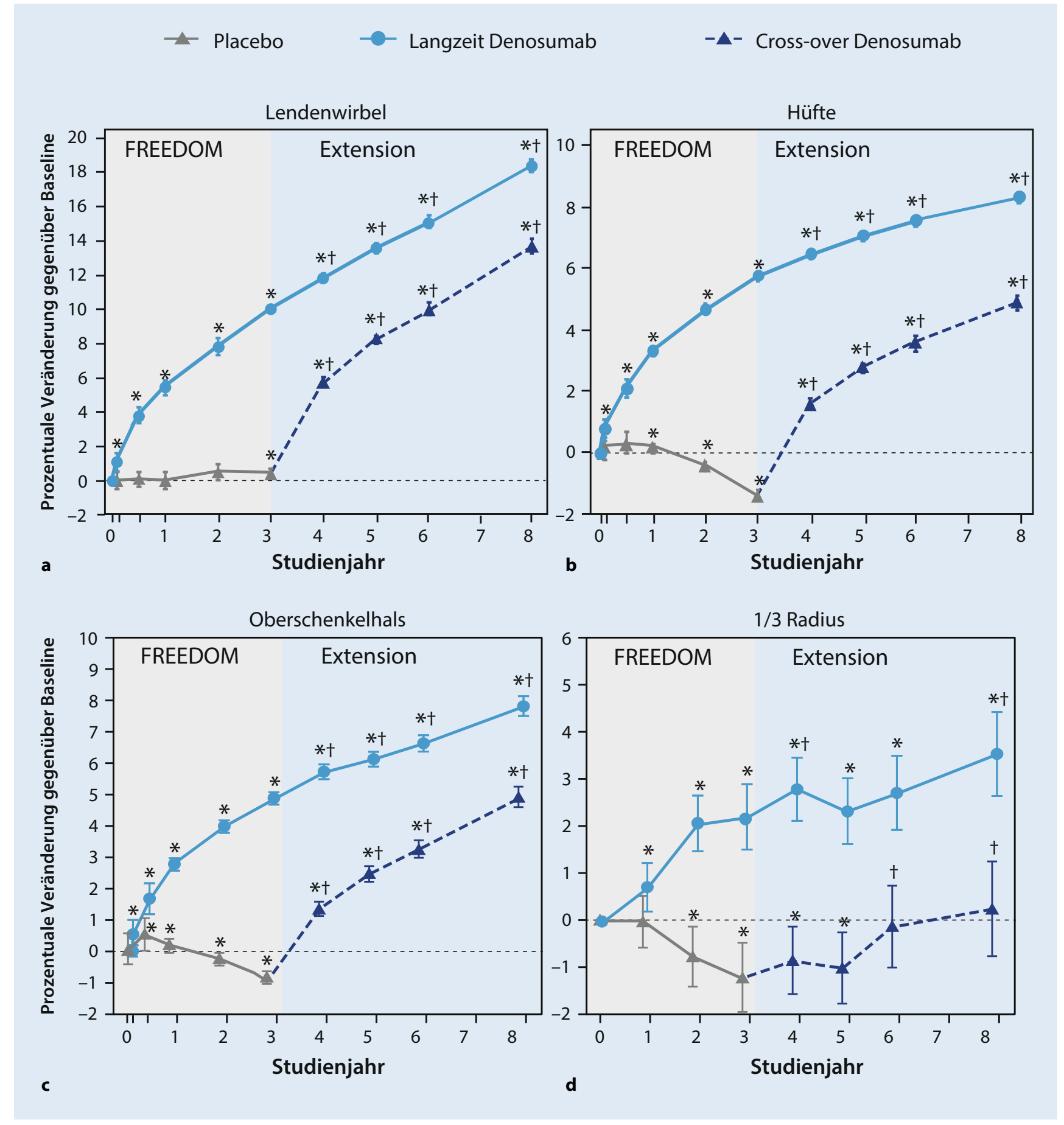

Abb. $3 \Delta$ Veränderung der Knochenmineraldichte in konsekutiven DXA-Messungen unter Denosumab. ${ }^{*} p<0,05$ verglichen mit FREEDOM-Baseline; $\uparrow p<0,05$ verglichen mit Extension-Baseline (Mit Genehmigung aus [5]. $\odot$ The Author(s) 2015 http:// creativecommons.org/licenses/by-nc/4.0/. Diese Abbildung fällt nicht unter die Creative Commons CC BY-Lizenz dieser Publikation)

einer verminderten Knochendichte hat der Sklerostinantikörper die Knochendichte innerhalb von zwölf Monaten an der Lendenwirbelsäule um $13 \%$ und an der Hüfte um $6 \%$ verbessert [8].
Nach 12 Monaten Romosozumab wurde die Studie mit einem oralen Bisphosphonat (Alendronat) weiter geführt. In der ARCH-Studie wurde Romosozumab im Vergleich zu wöchentlichem oralem Alendronat bei postmenopausa- len Frauen mit einem sehr hohen Frakturrisiko (herabgesetzte Knochendichte und prävalente Frakturen) untersucht (- Abb. 4 und 5; [9]).

Sowohl in der placebokontrollierten osteoporotischen Population als auch in 


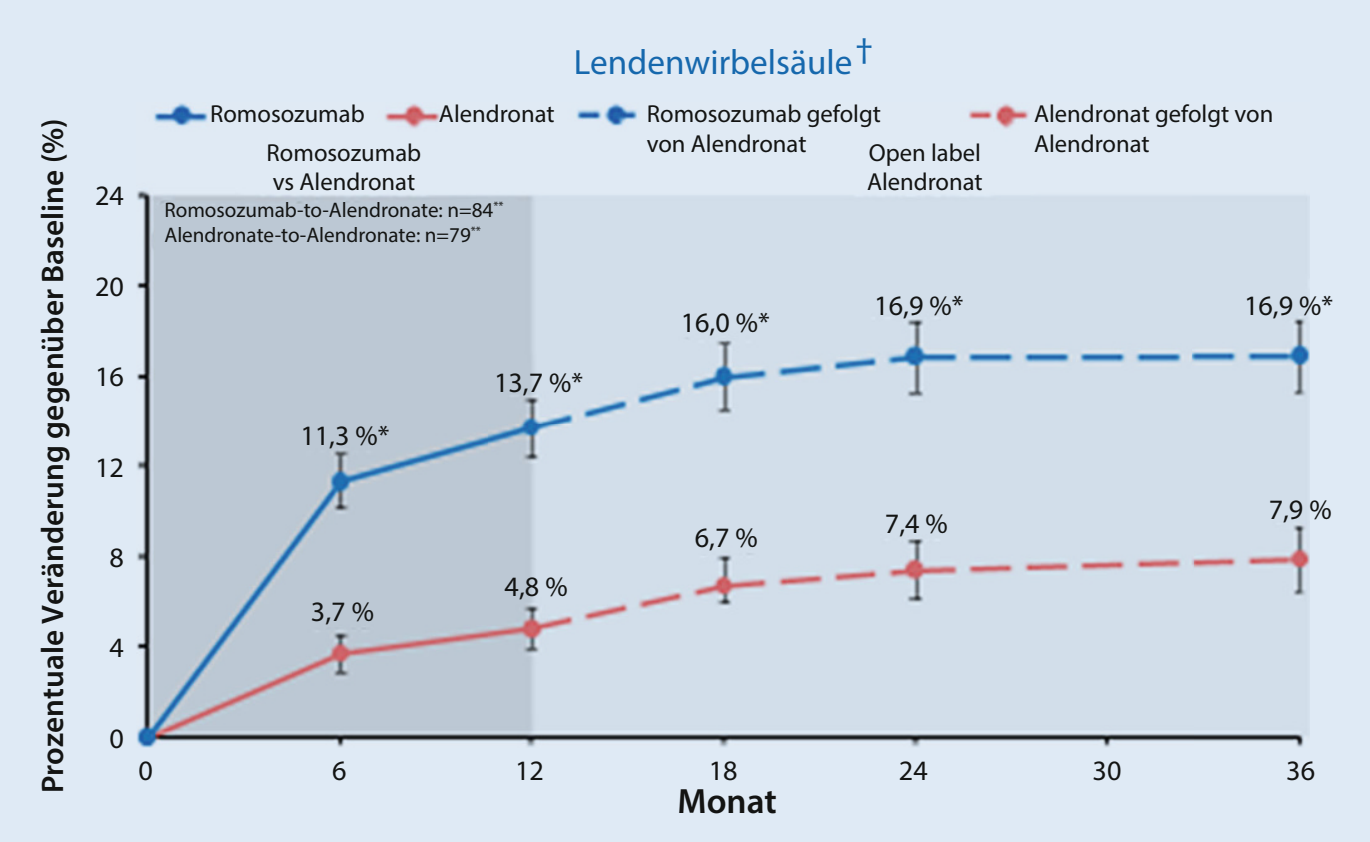

Abb. $4 \varangle$ Veränderungen der Knochendichte unter Romosozumab mit anschließender Konsolidierung mit oralem Alendronat. $p<0.001$ vs. baseline. (Mod. nach [9])

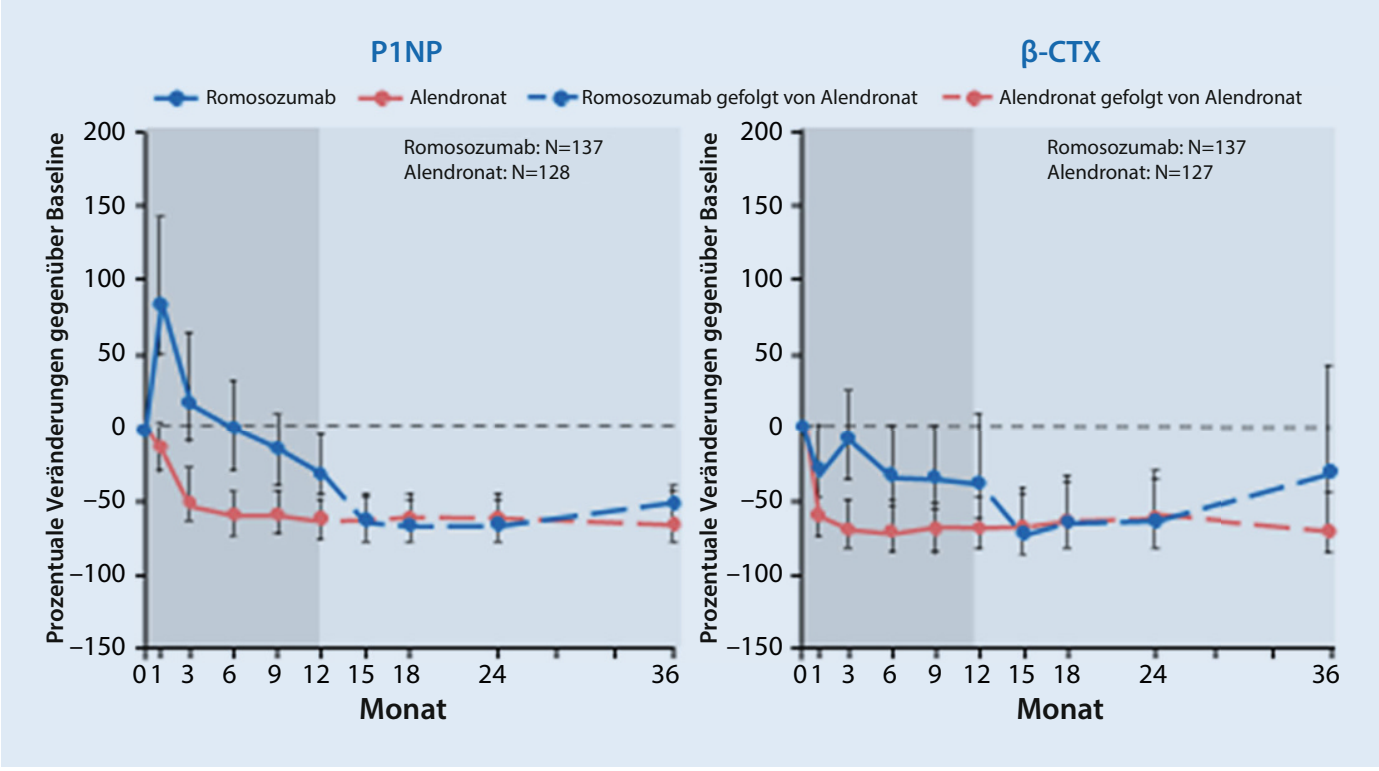

Abb. $5<$ Vergleich der Knochenstoffwechselmarker unter Therapie mit Romosozumab und Alendronat. (Mod. nach [9])

der Hochrisikopopulation kam es unter Romosozumab $\mathrm{zu}$ einer raschen und anhaltenden Reduktion von osteoporotischen Fragilitätsfrakturen (vertebral, nonvertebral, Hüfte).

Das Profil der Knochenstoffwechselmarker zeigt unter diesem Antikörper einen im Vergleich $\mathrm{zu}$ anderen Therapieoptionen rascheren Verlauf. Marker für Osteoblasten (P1NP) steigen rasch an und fallen im Verlauf wieder ab. Im Gegensatz dazu sinken die Marker für
Osteoklasten ( $\beta$-CTX) ab und verbleiben auf einem niedrigeren Niveau.

\section{Indikation und Erstattung}

Romosozumabwurde im Dezember 2019 von der European Medicines Agency (EMA) zur Behandlung von Frauen mit schwerer postmenopausaler Osteoporose (geringe Knochenmineraldichte und prävalente Fragilitätsfrakturen) und einem hohen Risiko für weitere Frakturen zugelassen. Es gibt einen Warnhinweis für Patientinnen mit einem Myokardinfarkt oder einem Insult in der Anamnese, da es in einer Studie zu einer numerischen Erhöhung dieser Ereignisse gekommen ist.

In Österreich ist das Medikament derzeit in der roten Box, es laufen aber bereits die Verhandlungen mit den Kostenträgern. Romosozumab kann derzeit nur in Ausnahmefällen in Spezialambulanzen verschrieben werden. 


\begin{tabular}{|c|c|}
\hline \multicolumn{2}{|r|}{ Praxis } \\
\hline \multicolumn{2}{|c|}{$\begin{array}{l}\text { Osteoporose ist in Österreich eine } \\
\text { Erkrankung mit hoher Prävalenz und } \\
\text { ökonomischer Belastung. } \\
\text { - Denosumab ist ein Antikörper mit } \\
\text { einem antikatabolen/antiresorptiven } \\
\text { Wirkmechanismus und verifizierter } \\
\text { Langzeit-Effizienz, bei Absetzen soll } \\
\text { eine konsolidierende Bisphosphonat- } \\
\text { therapie angeschlossen werden. } \\
\text { Romosozumab steht als Vertreter } \\
\text { einer anabolen Medikation bei } \\
\text { postmenopausaler Osteoporose mit } \\
\text { hohem Frakturrisiko ante portas. }\end{array}$} \\
\hline \multicolumn{2}{|c|}{ Korrespondenzadresse } \\
\hline & $\begin{array}{l}\text { iv.-Doz. Dr. } \\
\text { ristian Muschitz } \\
\text { Medizinische Abteilung, } \\
\text { ankenhaus der } \\
\text { rmherzigen Schwestern } \\
\text { en } \\
\text { umpergasse 13, } 1060 \text { Wier } \\
\text { terreich } \\
\text { ristian.muschitz@ } \\
\text { eduniwien.ac.at }\end{array}$ \\
\hline
\end{tabular}

Funding. Open access funding provided by Medical University of Vienna.

\section{Einhaltung ethischer Richtlinien}

Interessenkonflikt. C. Muschitz gibt an, dass kein Interessenkonflikt besteht.

Für diesen Beitrag wurden von den Autoren keine Studien an Menschen oder Tieren durchgeführt. Für die aufgeführten Studien gelten die jeweils dort angegebenen ethischen Richtlinien.

Open Access. Dieser Artikel wird unter der Creative Commons Namensnennung 4.0 International Lizenz veröffentlicht, welche die Nutzung, Vervielfältigung, Bearbeitung, Verbreitung und Wiedergabe in jeglichem Medium und Format erlaubt, sofern Sie den/die ursprünglichen Autor(en) und die Quelle ordnungsgemäß nennen, einen Link zur Creative Commons Lizenz beifügen und angeben, ob Änderungen vorgenommen wurden.

Die in diesem Artikel enthaltenen Bilder und sonstiges Drittmaterial unterliegen ebenfalls der genannten Creative Commons Lizenz, sofern sich aus der Abbildungslegende nichts anderes ergibt. Sofern das betreffende Material nicht unter der genannten Creative Commons Lizenz steht und die betreffende Handlung nicht nach gesetzlichen Vorschriften erlaubt ist, ist für die oben aufgeführten Weiterverwendungen des Materials die Einwilligung des jeweiligen Rechteinhabers einzuholen.
Weitere Details zur Lizenz entnehmen Sie bitte der Lizenzinformation auf http://creativecommons.org/ licenses/by/4.0/deed.de.

\section{Literatur}

1. Gesundheit Österreich GmbH. GÖG 2020 - Bericht über Fragilitätsfrakturen in Österreich im Jahr 2018.https://goeg.at/

2. Österreichische Leitlinie zur Behandlung der Osteoporose - veröffentlicht im Herbst 2017. www.arzneiundvernunft.at

3. Dempster DW, Brown JP, Fahrleitner-Pammer A Kendler D, Rizzo S, Valter I, Wagman RB, Yin X, Yue SV, Boivin G (2018) Effects of long-term denosumab on bone histomorphometry and mineralization in women with postmenopausal osteoporosis. J Clin Endocrinol Metab 103(7):2498-2509. https://doi.org/10.1210/jc. 2017-02669

4. Tsourdi E, Makras P, Rachner TD, Polyzos S, Rauner M, Mandanas S, Hofbauer LC, Anastasilakis AD (2019) Denosumab effects on bone density and turnover in postmenopausal women with low bone mass with or without previous treatment. Bone 120:44-49. https://doi.org/10.1016/j.bone. 2018.10.001

5. Papapoulos S, Lippuner K, Roux C et al (2015) The effect of 8 or 5 years of denosumab treatment in postmenopausal women with osteoporosis: results from the FREEDOM extension study. Osteoporos Int 26:2773-2783. https://doi.org/10. 1007/s00198-015-3234-7

6. Homepage der Österreichischen Gesellschaft für Knochen und Mineralstoffwechsel. www.oegkm. at

7. Fixen C, Tunoa J (2021) Romosozumab: a review of efficacy, safety, and cardiovascular risk. Curr Osteoporos Rep 19(1):15-22. https://doi.org/10. 1007/s11914-020-00652-w

8. Cosman F, Crittenden DB, Adachi JD, Binkley N, Czerwinski E, Ferrari S, Hofbauer LC, Lau E, Lewiecki EM, Miyauchi A, Zerbini CA, Milmont CE, Chen L, Maddox J, Meisner PD, Libanati C, Grauer A (2016) Romosozumab treatment in postmenopausal women with osteoporosis. N Engl J Med 375(16):1532-1543. https://doi.org/ 10.1056/NEJMoa1607948

9. Saag KG, Petersen J, Brandi ML, Karaplis AC, Lorentzon M, Thomas T, Maddox J, Fan M, Meisner PD, Grauer A (2017) Romosozumab or alendronate for fracture prevention in women with osteoporosis. N Engl J Med 377(15):1417-1427. https://doi.org/10.1056/NEJMoa1708322

Hinweis des Verlags. Der Verlag bleibt in Hinblick auf geografische Zuordnungen und Gebietsbezeichnungen in veröffentlichten Karten und Institutsadressen neutral.

\section{Telemedizin}

Marx, Gernot, Rossaint, Rolf, Marx, Nikolaus (Hrsg.)

2021, XVI, 502 S. 98 Abb., 79,99 EUR ISBN 978-3-662-60610-0

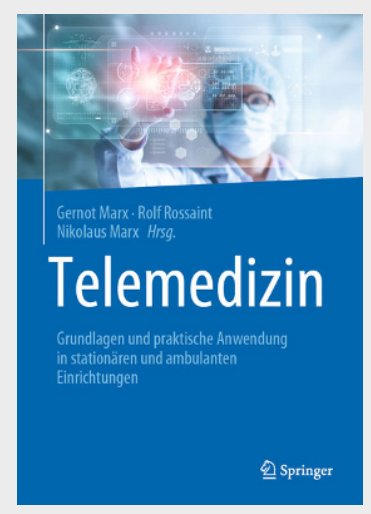

Grundlagen und praktische Anwendung in stationären und ambulanten Einrichtungen:

- Chancen, Risiken und rechtliche Besonderheiten der Telemedizin

- Mit zahlreichen Anwendungsbeispielen

- Mit Blick in die EU und Nachbarländer

Das Buch gibt einen umfassenden Überblick über den aktuellen Stand und die Entwicklungen im Bereich der Telemedizin in Deutschland. Das Herausgeberteam aus einem der führenden Telemedizinzentren Deutschlands und ein interdisziplinäres Autorenteam liefern wesentliche Grundlagen sowie zahlreiche Anwendungsbeispiele aus dem stationären und ambulanten Bereich. Zudem werden Chancen und Risiken der Telemedizin erörtert, so dass der Leser gut für die Zukunft in Klinik oder Praxis gerüstet ist. Ein Grundlagen- und Nachschlagewerk für alle Ärzte, die sich für digitale Zukunft im Gesundheitswesen wappnen und ihre Patienten optimal versorgen möchten. 